\title{
Using the Set Equality Method (SEM) to Determine the Validity of Categorical Syllogisms
}

\author{
Osvaldo Skliar* Sherry Gapper ${ }^{\dagger} \quad$ Ricardo E. Monge G $^{\ddagger}$
}

February 12, 2022

\begin{abstract}
A description is provided of a method - the Set Equality Method (SEM) - to determine the validity, or lack of validity, of each categorical syllogism. A justification is given for the presentation of a new method to solve a problem which has already been solved using different approaches. First, the SEM assigns an equality of certain sets (or two of those equalities in specific cases as will be indicated) to each of the categorical propositions composing each syllogism considered - that is, to each of the two premises and to the conclusion. Each syllogism considered is valid if and only if a) it is possible to select one of those equalities corresponding to one of the premises such that one of its members is a certain set and the other of those equalities corresponding to the other premise such that one of its members is a subset of the set mentioned, and b) it is possible to deduce an equality corresponding to the conclusion of the two equalities corresponding to the premises. In some cases, as will be specified, it is possible to provide a second test for the validity of a syllogism whose validity was already proven, thus providing information about the logical form of categorical syllogisms.
\end{abstract}

Keywords: categorical syllogisms, categorical propositions, set theory, set equalities

Mathematics Subject Classification 2020: 03B05, 03B10, 97E30, 97E60

\footnotetext{
${ }^{*}$ Universidad Nacional, Costa Rica. E-mail: osvaldoskliar@gmail.com. https://orcid.org/0000-0002-8321-3858.

†Universidad Nacional, Costa Rica. E-mail: sherry.gapper.morrow@una.ac.cr. https://orcid.org/0000-0003-4920-6977.

$\ddagger$ Universidad CENFOTEC, Costa Rica. E-mail: rmonge@ucenfotec.ac.cr. https://orcid.org/0000-0002-4321-5410.
} 


\section{Introduction}

From historical and educational standpoints, forms of reasoning known as syllogisms, categorical syllogisms in particular, have played an important role in logic.

Determining the validity, or lack of validity, of any categorical syllogism is not an open problem. Hence, for example, one of the methods which may be used to solve that problem is presented in chapter 16, "Syllogisms", of W. V. Quines' Method of Logic [1]. Other methods used with that same objective have been presented, for example, in [2], [3], [4], [5], [6], and [7]. The authors of this article have introduced two more of those methods: the Inclusion Diagrams Method (IDM) [8] and the Membership Table Method (MTM) [9].

Presenting another method to solve this problem is justifiable if a) that new method is significantly different from previously known methods used to reach that objective, because it would be fruitful to focus on a problem from different standpoints, and b) it can be more easily understood and applied than other existing methods.

The objective of this article is to present a new method - the Set Equality Method (SEM) - to determine the validity, or lack of validity, of any categorical syllogism.

A clear understanding of this article requires only a basic knowledge of a) certain notions of logic such as those of reasoning, deduction (or inference) and statement (that is, proposition or sentence), and b) set theory. For an introduction to logic, see, for example: [10], [11], [12], [13], and [14]; and for set theory, see, for example, [15], [16], [17], and [18].

\section{Review of Basic Notions of Set Theory}

When using set theory to address a given topic, first it is fitting to specify what the universal set $\mathbb{U}$ is; that is the universe of discourse, the set to which all the elements that may be considered belong. Only elements belonging to $\mathbb{U}$ can belong to the different sets characterized within its framework. The empty set - symbolized as $\varnothing$ - characterized within the framework of that $\mathbb{U}$, is the set to which any element belonging to $\mathbb{U}$ does not belong. Thus, there is no element belonging to $\varnothing$.

In sections 2 and 3 uppercase letters such as $F, G$ and $H$ are used to denominate each of the sets characterized within the framework of some $\mathbb{U}$. The denominations of the sets $C_{1}, C_{2}$ and $C_{3}$ are reserved for a special use to be discussed in section 4 .

The complement, or complementary set, of the set $F$ is represented by the symbol $\stackrel{+}{F}$. Recall that all of the elements belonging to the $\mathbb{U}$ considered that do not belong to $F$ belong to the complement of any set $F$. Given this characterization of $\vec{F}$ - that is, of the complement of any set $F$ - the following results are easily deduced: 


\section{1. $\stackrel{+}{\mathbb{U}}=\varnothing$}

Given that all the elements belonging to $\mathbb{U}$ and not belonging to a specific set belong to the complement of that set, and that all of the elements considered when addressing a given topic belong to $\mathbb{U}$, it is evident that no element belongs to $\stackrel{+}{\mathbb{U}}$. Therefore, $\overrightarrow{\mathbb{U}}=\varnothing$.

$$
\text { 2. } F \cap \stackrel{+}{F}=\varnothing
$$

Written out: Given that all the elements belonging to $\mathbb{U}$ that do not belong to $F$ belong to the complement of $F$ (that is, $\vec{F}$ ), it is evident that $F$ and $\stackrel{+}{F}$ have no elements in common (that is, no element belongs to both $F$ and $\vec{F}$. Therefore, the intersection of $F$ and $\stackrel{\vec{F}}{F}$ is equal to the empty set. (Recall that all of the elements that belong both to one of those two sets and to the other belong to the intersection of two sets.) In particular, $\mathbb{U} \cap \varnothing=\varnothing$.

\section{3. $F \cup \vec{F}=\mathbb{U}$}

Written out: Given that all the elements belonging to $\mathbb{U}$ that do not belong to $F$ belong to the complement of $F$ (that is, $\vec{F}$ ), it is evident that the union of $F$ and $\vec{F}$ is equal to the universal set $\mathbb{U}$. (Recall that any element belonging to either of those two sets, or to both, belongs to the union of two sets.) In particular, $\mathbb{U} \cup \varnothing=\mathbb{U}$.

If any element belonging to the set $F$ also belongs to a set $G$, then $F$ is considered a subset of $G$. The relation between $F$ and $G$ can be established by the following equality:

$$
F=S_{a}(G)
$$

In the right-hand member of the above equality, the uppercase letter $S$ refers to a $S$ ubset. The uppercase $G$ placed in parentheses after $S_{a}$ specifies that it refers to a subset of set $G$. The subscript $a$ of $S$ indicates that $S_{a}$ is one of the different subsets of the set $G$. Recall that if $n$ elements, for $n=0,1,2,3, \ldots$, belong to any set $G$, there are $2^{n}$ subsets of $G$. (The case $n=0$ is that which corresponds to $G=\varnothing$.) Another lowercase letter, such as $b, c, d, e$ or $f$, could have been selected instead of $a$. In this article, however, the lowercase letters $x$ and $y$ are symbols reserved for special subscripts whose nature is specified in sección 5 .

Subsets of different sets are referred to by using different subscripts of $S$. Consider, for example, the following equality:

$$
S_{a}(F)=S_{b}(G)
$$

The left-hand member of the above equality is a particular subset of $F$ and the right-hand member of that equality is a particular subset of $G$. Of course, 
any subset of any set is also a set. The equality of $S_{a}(F)$ and $S_{b}(G)$ specifies that, considered as sets, these subsets are equal, or in other words, they are the same set. The same elements belong to both.

A subset of any subset of any set $H$ is also a specific subset of $H$.

$$
S_{b}\left(S_{a}(H)\right)=S_{c}(H)
$$

Recall that the equality $F=S_{a}(G)$ is equivalent, from the standpoint of logic, to $\stackrel{+}{G}=S_{b}(\stackrel{+}{F})$; that is, both equalities have the same truth value. In other words, the statement " $F$ is equal to a certain subset of $G$ " has the same truth value as the statement " $\stackrel{+}{G}$ is equal to a certain subset of $\stackrel{+}{F}$ ". That is, each of the statements is true if and only if the other is also true. Therefore, if one of them is not true (i.e., false), then the other one is also false.

Consideration is given below to some sets that make it possible to note how, in certain cases, the "laws" of set theory provided above are verified.

Take, for example, a universal set $\mathbb{U}$ to which the natural numbers $1,2,3$, 4, 5, 6, 7, 8, 9 and 10 belong:

$$
\{\mathbb{U}=1,2,3,4,5,6,7,8,9,10\}
$$

Let $F, G$ and $H$ be the following sets characterized within the framework of that universal set $\mathbb{U}$.

$$
\begin{aligned}
& F=\{2,7\} \\
& G=\{2,5,7\} \\
& H=\{1,2,4,5,6,7,9\}
\end{aligned}
$$

Therefore,

$$
\begin{aligned}
& \stackrel{+}{F}=\{1,3,4,5,6,8,9,10\} \\
& \stackrel{+}{G}=\{1,3,4,6,8,9,10\} \\
& \stackrel{+}{H}=\{3,8,10\} \\
& \mathbb{U}=F \cup \stackrel{+}{F}=G \cup \stackrel{+}{G}=H \cup \stackrel{+}{H}=\mathbb{U} \cup \varnothing \\
& \varnothing=F \cap \stackrel{+}{F}=G \cap \stackrel{+}{G}=H \cap \stackrel{+}{H}=\mathbb{U} \cap \varnothing \\
& F=S_{a}(G) ; \quad G=S_{b}(H) ; \quad F=S_{a}\left(S_{b}(H)\right)=S_{c}(H) \\
& \stackrel{+}{G}=S_{d}(\stackrel{+}{F}) ; \quad \stackrel{+}{H}=S_{e}(G) ; \quad \stackrel{+}{H}=S_{f}(\stackrel{+}{F})
\end{aligned}
$$


The relation of complementation between two sets is symmetrical: The complement of a set $F$ is the set $\vec{F}$ if and only if the complement of the set $\vec{F}$ is the set $F$. Therefore:

$$
\stackrel{+}{F}=F
$$

Written out: The operation of "double complementation" of any set $F$ generates, as a result, the set $F$.

Below the symbols $F$ and $G$ will be used to denominate sets different from those in the above example. Consider this equality:

$$
F=S_{a}(\bar{G})
$$

The above equality is equivalent, from the standpoint of logic, to the equality below:

$$
\stackrel{+}{G}=S_{b}(\stackrel{+}{F})
$$

Given that $\stackrel{+}{G}=G$, the following two equalities are equivalent:

$$
F=S_{a}(\stackrel{+}{G}) ; \quad G=S_{b}(\stackrel{+}{F})
$$

This result can be expressed also as follows: A set $F$ is a subset of $\stackrel{t}{G}$ if and only if $G$ is a subset of $\stackrel{+}{F}$.

\section{Categorical Propositions}

In this article, the same meaning is given to the terms "statement" and "proposition". In the literature devoted to logic, references to "categorical propositions" are found more frequently than to "categorical statements".

Categorical propositions are assertions (about sets) which affirm or negate that one set is totally or partially included in another.

There are four types of categorical propositions: 1. universal affirmative propositions; 2) universal negative propositions; 3 . particular affirmative propositions; and 4) particular negative propositions.

Consideration is given below to an example of each of these types of categorical propositions.

1) Example of a universal affirmative proposition:

All generals are brave.

According to the above proposition, each element belonging to a certain set $F$ also belongs to a certain set $G$. Those sets are:

$$
F \text { : generals; } \quad G \text { : brave persons }
$$


In general, universal affirmative propositions can be expressed as follows:

All $F$ are $G$.

Using the terminology of set theory, the above proposition can be expressed by either of the following two equalities, considered equivalent from the standpoint of logic:

$$
F=S_{a}(G) ; \quad \stackrel{+}{G}=S_{b}(\stackrel{+}{F})
$$

2) Example of a negative universal negative proposition:

No general is brave.

In general, universal negative propositions can be expressed as follows:

No $F$ is $G$.

According to the above proposition, no element belonging to the set denominated $F$ belongs to the set denominated $G$. Therefore, every element belonging to $F$ also belongs to $\vec{G}$. Using the terminology of set theory, the preceding proposition can be expressed by either of the following two equalities, equivalent from the standpoint of logic:

$$
\left.F=S_{a}(\vec{G}) ; \quad G=S_{b} \vec{F}\right)
$$

3) Example of a particular affirmative proposition:

Some generals are brave.

In general, particular affirmative propositions can be expressed as follows:

Some $F$ are $G$.

According to the above proposition, some elements belonging to $F$ are also some of the elements belonging to $G$. Using the terminology of set theory, the preceding proposition can be expressed by the following equality:

$$
S_{a}(F)=S_{b}(G)
$$

4) Example of a particular negative proposition:

Some generals are not brave.

In general, particular negative propositions can be expressed as follows: 
Some $F$ are not $G$.

According to the above proposition, some elements belonging to $F$ are also some of the elements belonging to $\vec{G}$ ). Using the terminology of set theory, the preceding proposition can be expressed by the following equality:

$$
S_{a}(F)=S_{b}(\stackrel{+}{G})
$$

\section{Characterization of Categorical Syllogisms}

A categorical syllogism is a type of reasoning composed of three categorical propositions such that the third of them (denominated conclusion), can be deduced, if the syllogism is valid, from the first two (denominated premises).

An example of a categorical syllogism is presented below. In this article the first premise, the second premise and the conclusion of any categorical syllogism are denominated, respectively, $s_{1}, s_{2}$ and $s_{3}$. The letter "s" was used because it is the first letter of the word "statement".

$s_{1}$ : All engineers are pragmatic.

$s_{2}$ : Some engineers are wealthy.

$\therefore s_{3}$ : Some wealthy persons are pragmatic.

The symbol $\therefore$ means "therefore".

The term for the predicate of the conclusion - in this case "pragmatic (persons)" is denominated the "major term" in any categorical syllogism and corresponds to the set denominated $C_{3}$ in this article. The term of the subject of the conclusion - in this case "wealthy persons" is denominated the "minor term" in any categorical syllogism and corresponds to a set denominated $C_{1}$ in this article. The premise in which the major term is contained is denominated major premise. Here that is systematically the first premise $s_{1}$ of each categorical syllogism considered. The premise in which the minor term is contained is denominated the minor premise. Here it is systematically the second premise $s_{2}$ of each categorical syllogism considered. There is a term which is not contained in the conclusion but it is contained in both premises. That term, "engineers" in this case, is called the "middle term" and corresponds to the set denominated $C_{2}$ in this article.

For the first example of a categorical syllogism presented below, attention is again given to what the sets $C_{1}, C_{2}$, and $C_{3}$ are:

$$
C_{1} \text { : wealthy persons } \quad C_{2} \text { : engineers } \quad C_{3} \text { : pragmatic persons }
$$

All of the categorical syllogisms of the same type as the syllogism considered can be expressed in the following way:

$$
s_{1}: \text { All } C_{2} \text { are } C_{3} \text {. }
$$


$s_{2}$ : Some $C_{2}$ are $C_{1}$.

$\therefore s_{3}$ : Some $C_{1}$ are $C_{3}$.

Consider another example of a categorical syllogism:

$s_{1}$ : No intellectuals are superstitious.

$s_{2}$ : Some French persons are intellectuals.

$\therefore s_{3}$ : Some French persons are not superstitious.

In this syllogism, the sets $C_{1}, C_{2}$ and $C_{3}$ are the following:

$$
C_{1} \text { : French persons } \quad C_{2} \text { : intellectuals } \quad C_{3} \text { : superstitious per- }
$$$$
\text { sons }
$$

All of the categorial syllogisms of the same type as this last syllogism can be expressed in the following way:

$s_{1}:$ No $C_{2}$ are $C_{3}$.

$s_{2}$ : Some $C_{1}$ are $C_{2}$.

$\therefore s_{3}$ : Some $C_{1}$ are not $C_{3}$.

\section{Using the SEM to Determine the Validity of Categorical Syllogisms}

Any categorical syllogism is considered valid if and only if its conclusion can be deduced from its premises.

When symbolizing each premise of a categorical syllogism as an equality of two sets, in the references to subsets, which are also sets, use is made of the lowercase letters $a, b, c, d, e$ and $f$, as subscripts of the symbol $S$ for $S$ ubset (as indicated in section 2). On the other hand, when symbolizing the conclusion of each categorical syllogism as an equality of two sets, in the references to subsets, use is made of the lowercase letters $x$ and $y$ as subscripts of $S$. These subscripts $-x$ and $y$ - can be replaced, if pertinent, by lowercase letters such as $a, b, c, d, e$ and $f$. Consider, for example, one possible conclusion of a categorical syllogism symbolized as the following equality of sets: $F=S_{x}(H)$. Let us admit that from the premises of the categorical syllogism considered, the following set equality has been deduced: $F=S_{e}(H)$. Note that if in the former set equality $x$ is replaced by $e$, that equality is identical to the latter equality; that is, both equalities are the same equality. In this case, given that the conclusion of the syllogism considered has been deduced from its two premises, that syllogism is considered valid.

Consider another possible conclusion of a categorical syllogism symbolized as the following equality of two subsets: $S_{x}(F)=S_{y}(H)$. Admit that from the premises of the categorical syllogism considered the following equality has been deduced: $S_{c}(F)=S_{e}(H)$. Note that if in the first of the latter two equalities $x$ is replaced by $c$, and $y$ by $e$, that first equality is identical to the second 
one; that is, both equations are the same equality. In this case, given that the conclusion of the syllogism considered has been deduced from its premises, that syllogism is valid.

To apply the SEM, it must first be determined whether it is possible to select, from the equalities of sets corresponding to the premises of the syllogism considered, an equality one of whose members is $C_{2}$ and another equality one of whose members is a subset of $C_{2}$. Suppose a) that one of those two equalities can be selected, and b) that from them it is possible to deduce (as specified below for each of the examples of categorical syllogisms examined in this section) a set equality corresponding to the conclusion of the syllogism considered. Both if supposition a) is false, and if supposition b) is false, it must be determined whether from the set of equalities corresponding to the premises of the syllogism considered, one of them can be selected such that one of its members is $\stackrel{C}{C}_{2}$ and another of its members is a subset of $\vec{C}_{2}$. Suppose that c) those two set equalities can be selected, and that d) from them it is possible to deduce a set equality corresponding to the conclusion of the syllogism considered. In this case, that syllogism is deemed valid. Both if supposition c) is false, and if supposition d) is false, the syllogism considered is not valid.

Even if it is proven that a) and b) are true, in which case the syllogism considered is valid, it is worth determining whether suppositions c) and d) are also valid, in which case another proof of validity of that syllogism has been obtained.

The SEM is applied below to 16 examples of categorical syllogisms. The first six were taken from [1].

In each of those examples the following is provided:

1) the categorical syllogism considered, expressed in natural language;

2) a specification of what the sets $C_{1}, C_{2}$, and $C_{3}$ are;

3) an expression of the syllogism considered whose minor term, middle term and major term were replaced by $C_{1}, C_{2}$, and $C_{3}$, respectively;

4) the set equality (or if pertinent, both of the respective set equalities) corresponding to each of its statements (to each of the premises and to the conclusion); and

5) the application of the SEM to the syllogism considered.

Example 1

$s_{1}:$ All men are mortal.

$s_{2}:$ All Greeks are men.

$\therefore s_{3}$ : All Greeks are mortal. 


$$
\begin{aligned}
& C_{1} \text { : Greeks } \quad C_{2} \text { : men } \quad C_{3} \text { : mortal beings } \\
& s_{1}: \text { All } C_{2} \text { are } C_{3} . \quad C_{2}=S_{a}\left(C_{3}\right) \quad(1) ; \quad \overleftarrow{C}_{3}=S_{b}\left(\overleftarrow{C}_{2}\right) \\
& s_{2}: \text { All } C_{1} \text { are } C_{2} . \quad C_{1}=S_{c}\left(C_{2}\right) \quad(2) ; \quad \overleftarrow{C}_{2}=S_{a}\left(\overleftarrow{C}_{1}\right) \\
& \therefore s_{3} \text { : All } C_{1} \text { are } C_{3} . \quad C_{1}=S_{x}\left(C_{3}\right) \quad(3) ; \quad \bar{C}_{3}=S_{y}\left(\overleftarrow{C}_{1}\right)
\end{aligned}
$$

Set equality (1) is selected because it includes $C_{2}$ as one of its members, and set equality (2) is selected because it includes a subset of $C_{2}$ as one of its members.

$$
\begin{aligned}
& C_{2}=S_{a}\left(C_{3}\right) \\
& C_{1}=S_{c}\left(C_{2}\right)
\end{aligned}
$$

In (2), $C_{2}$ is replaced by the right-hand member of equality (1):

$$
C_{1}=S_{c}\left(S_{a}\left(C_{3}\right)\right)=S_{e}\left(C_{3}\right)
$$

From (1) and (2), the following equality was deduced:

$$
C_{1}=S_{e}\left(C_{3}\right)
$$

If in (3) the subscript $x$ is replaced by $e$, equality (3) - an expression of the conclusion - is identical to equality (4), which was deduced from equalities (1) and (2), expressions of the first and second premises, respectively. Therefore, the syllogism considered is valid.

Another proof of validity of that syllogism is provided below.

Set equality $\left(1^{\prime}\right)$ is selected because it includes a subset of $\vec{C}_{2}$ as one of its members, and $\left(2^{\prime}\right)$ is selected because it includes $\vec{C}_{2}$ as one of its members.

$$
\begin{aligned}
& \overleftarrow{C}_{3}=S_{b}\left(\overleftarrow{C}_{2}\right) \\
& \stackrel{+}{C}_{2}=S_{d}\left(\stackrel{C}{C}_{1}\right)
\end{aligned}
$$

In $\left(1^{\prime}\right), \stackrel{+}{C}_{2}$ is replaced by the right-hand member of equality $\left(2^{\prime}\right)$ :

$$
\stackrel{\mathrm{C}}{3}_{3}=S_{b}\left(S_{d}\left(\overleftarrow{C}_{1}\right)\right)=S_{f}\left(\overleftarrow{C}_{1}\right)
$$

From $\left(1^{\prime}\right)$ and $\left(2^{\prime}\right)$ the following equality was deduced:

$$
\stackrel{+}{C}_{3}=S_{f}\left(\stackrel{+}{C}_{1}\right)
$$

If in $\left(3^{\prime}\right)$ the subscript $y$ is replaced by $f$, equality $\left(3^{\prime}\right)$ - an expression of the conclusion - is identical to equality $\left(4^{\prime}\right)$, which was deduced from equalities $\left(1^{\prime}\right)$ and $\left(2^{\prime}\right)$, expressions of the first and second premises, respectively. Therefore, another proof of the validity of the syllogism considered has been obtained. 


\section{Example 2}

$s_{1}$ : No men are perfect.

$s_{2}$ : All Greeks are men.

$\therefore s_{3}$ : No Greeks are perfect.

$$
\begin{aligned}
& C_{1} \text { : Greeks } \quad C_{2} \text { : men } \quad C_{3} \text { : perfect beings } \\
& s_{1} \text { : No } C_{2} \text { are } C_{3} . \quad C_{2}=S_{a}\left(\stackrel{C}{C}_{3}\right) \quad(1) ; \quad C_{3}=S_{b}\left(\overleftrightarrow{C}_{2}\right) \\
& s_{2}: \text { All } C_{1} \text { are } C_{2} . \quad C_{1}=S_{c}\left(C_{2}\right) \quad(2) ; \quad \stackrel{+}{C}_{2}=S_{d}\left(\stackrel{+}{C}_{1}\right) \\
& \therefore s_{3} \text { : No } C_{1} \text { are } C_{3} . \quad C_{1}=S_{x}\left(\stackrel{+}{C}_{3}\right) \quad(3) ; \quad C_{3}=S_{y}\left(\stackrel{+}{C}_{1}\right)
\end{aligned}
$$

Set equality (1) is selected because it includes $C_{2}$ as one of its members, and set equality (2) is selected because it includes a subset of $C_{2}$ as one of its members.

$$
\begin{aligned}
& C_{2}=S_{a}\left(\overleftarrow{C}_{3}\right) \\
& C_{1}=S_{c}\left(C_{2}\right)
\end{aligned}
$$

In (2), $C_{2}$ is replaced by the right-hand member of equality (1):

$$
C_{1}=S_{c}\left(S_{a}\left(\overleftarrow{C}_{3}\right)\right)=S_{e}\left(\overleftarrow{C}_{3}\right)
$$

From (1) and (2), the following equality was deduced:

$$
C_{1}=S_{e}\left(\stackrel{+}{C}_{3}\right)
$$

If in (3) the subscript $x$ is replaced by $e$, equality (3) - an expression of the conclusion - is identical to equality (4), which was deduced from equalities (1) and (2), expressions of the first and second premises, respectively. Therefore, the syllogism considered is valid.

Another proof of validity of that syllogism is provided below.

Set equality $\left(1^{\prime}\right)$ is selected because it includes a subset of $\vec{C}_{2}$ as one of its members, and set equality $\left(2^{\prime}\right)$ is selected because it includes $\vec{C}_{2}$ as one of its members.

$$
\begin{aligned}
& \stackrel{+}{C}_{3}=S_{b}\left(\stackrel{+}{C}_{2}\right) \\
& \stackrel{+}{C}_{2}=S_{d}\left(\overleftarrow{C}_{1}\right)
\end{aligned}
$$

In $\left(1^{\prime}\right), \overleftrightarrow{C}_{2}$ is replaced by the right-hand member of equality $\left(2^{\prime}\right)$ :

$$
\stackrel{+}{C}_{3}=S_{b}\left(S_{d}\left(\bar{C}_{1}\right)\right)=S_{f}\left(\vec{C}_{1}\right)
$$

From $\left(1^{\prime}\right)$ and $\left(2^{\prime}\right)$ the following equality was deduced:

$$
\stackrel{+}{C}_{3}=S_{f}\left(\stackrel{C}{C}_{1}\right)
$$


If in $\left(3^{\prime}\right)$ the subscript $y$ is replaced by $f$, equality $\left(3^{\prime}\right)-$ an expression of the conclusion - is identical to equality $\left(4^{\prime}\right)$, which was deduced from equalities $\left(1^{\prime}\right)$ and $\left(2^{\prime}\right)$, expressions of the first and second premises, respectively. Therefore, another proof of the validity of the syllogism considered has been obtained.

\section{Example 3}

$s_{1}$ : All philosophers are wise.

$s_{2}$ : Some Greeks are philosophers.

$\therefore s_{3}$ : Some Greeks are wise.

$$
C_{1} \text { : Greeks } \quad C_{2} \text { : philosophers } \quad C_{3} \text { : wise persons }
$$

$$
\begin{aligned}
& s_{1}: \text { All } C_{2} \text { are } C_{3} . \quad C_{2}=S_{a}\left(C_{3}\right) \quad(1) ; \quad \stackrel{+}{C}_{3}=S_{b}\left(\stackrel{+}{C}_{2}\right) \\
& s_{2} \text { : Some } C_{1} \text { are } C_{2} . \quad S_{c}\left(C_{1}\right)=S_{d}\left(C_{2}\right) \\
& \therefore s_{3} \text { : Some } C_{1} \text { are } C_{3} . \quad S_{x}\left(C_{1}\right)=S_{y}\left(C_{3}\right)
\end{aligned}
$$

Set equality (1) is selected because it includes $C_{2}$ as one of its members, and set equality (2) is selected because it includes a subset of $C_{2}$ as one of its members.

$$
\begin{array}{r}
C_{2}=S_{a}\left(C_{3}\right) \\
S_{c}\left(C_{1}\right)=S_{d}\left(C_{2}\right)
\end{array}
$$

In (2), $C_{2}$ is replaced by the right-hand member of equality (1):

$$
S_{c}\left(C_{1}\right)=S_{d}\left(S_{a}\left(C_{3}\right)\right)=S_{e}\left(C_{3}\right)
$$

From (1) and (2), the following equality was deduced:

$$
S_{c}\left(C_{1}\right)=S_{e}\left(C_{3}\right)
$$

If in (3) subscripts $x$ and $y$ are replaced by $c$ and $e$, respectively, equality (3) - an expression of the conclusion - is identical to equality (4), which was deduced from equalities (1) and (2), expressions of the first and second premises, respectively. Therefore, the syllogism considered is valid.

\section{Example 4}

$s_{1}$ : No philosophers are wicked.

$s_{2}$ : Some Greeks are philosophers.

$\therefore s_{3}$ : Some Greeks are not wicked.

$$
\begin{aligned}
& C_{1} \text { : Greeks } \quad C_{2} \text { : philosophers } \quad C_{3} \text { : wicked persons } \\
& s_{1}: \text { No } C_{2} \text { are } C_{3} . \quad C_{2}=S_{a}\left(\vec{C}_{3}\right) \quad(1) ; \quad C_{3}=S_{b}\left(\vec{C}_{2}\right) \\
& s_{2} \text { : Some } C_{1} \text { are } C_{2} . \quad S_{c}\left(C_{1}\right)=S_{d}\left(C_{2}\right) \\
& \therefore s_{3} \text { : Some } C_{1} \text { are not } C_{3} . \quad S_{x}\left(C_{1}\right)=S_{y}\left(\stackrel{+}{C}_{3}\right)
\end{aligned}
$$


Set equality (1) is selected because it includes $C_{2}$ as one of its members, and set equality (2) is selected because it includes a subset of $C_{2}$ as one of its members.

$$
\begin{array}{r}
C_{2}=S_{a}\left(\dot{C}_{3}\right) \\
S_{c}\left(C_{1}\right)=S_{d}\left(C_{2}\right)
\end{array}
$$

In (2), $C_{2}$ is replaced by the right-hand member of equality (1):

$$
S_{c}\left(C_{1}\right)=S_{d}\left(S_{a}\left(\bar{C}_{3}\right)\right)=S_{e}\left(\bar{C}_{3}\right)
$$

From (1) and (2), the following equality was deduced:

$$
S_{c}\left(C_{1}\right)=S_{e}\left(\dot{C}_{3}\right)
$$

If in (3) subscripts $x$ and $y$ are replaced by $c$ and $e$, respectively, equality (3) - an expression of the conclusion - is identical to equality (4), which was deduced from equalities (1) and (2), expressions of the first and second premises, respectively. Therefore, the syllogism considered is valid.

\section{Example 5}

$s_{1}$ : All Greeks are men.

$s_{2}$ : Some mortals are not men.

$\therefore s_{3}$ : Some mortals are not Greeks.

$$
C_{1} \text { : mortal beings } \quad C_{2} \text { : men } \quad C_{3} \text { : Greeks }
$$

$$
\begin{aligned}
& s_{1}: \text { All } C_{3} \text { are } C_{2} . \quad C_{3}=S_{a}\left(C_{2}\right) \quad(1) ; \quad \stackrel{+}{C}_{2}=S_{b}\left(\mathbb{C}_{3}\right) \\
& s_{2} \text { : Some } C_{1} \text { are not } C_{2} . \quad S_{c}\left(C_{1}\right)=S_{d}\left(\overleftarrow{C}_{2}\right) \\
& \therefore s_{3} \text { : Some } C_{1} \text { are not } C_{3} . \quad S_{x}\left(C_{1}\right)=S_{y}\left(\stackrel{+}{C}_{3}\right)
\end{aligned}
$$

Set equality $\left(1^{\prime}\right)$ is selected because it includes $\vec{C}_{2}$ as one of its members, and set equality (2) is selected because it includes a subset of $\stackrel{十}{C}_{2}$ as one of its members.

$$
\begin{array}{r}
\stackrel{+}{C}_{2}=S_{b}\left(\stackrel{+}{C}_{3}\right) \\
S_{c}\left(C_{1}\right)=S_{d}\left(\stackrel{十}{C}_{2}\right)
\end{array}
$$

In $(2), \stackrel{C}{C}_{2}$ is replaced by the right-hand member of equality $\left(1^{\prime}\right)$ :

$$
S_{c}\left(C_{1}\right)=S_{d}\left(S_{b}\left(\overleftarrow{C}_{3}\right)\right)=S_{e}\left(\overleftarrow{C}_{3}\right)
$$

From $\left(1^{\prime}\right)$ and (2), the following equality was deduced:

$$
S_{c}\left(C_{1}\right)=S_{e}\left(\dot{C}_{3}\right)
$$


If in (3) subscripts $x$ and $y$ are replaced by $c$ and $e$, respectively, equality (3) - an expression of the conclusion - is identical to equality (4), which was deduced from equalities $\left(1^{\prime}\right)$ and (2), expressions of the first and second premises, respectively. Therefore, the syllogism considered is valid.

Example 6

$s_{1}$ : Some men are not Greeks.

$s_{2}$ : All men are mortal.

$\therefore s_{3}$ : Some mortals are not Greeks.

$$
\begin{aligned}
& C_{1} \text { : mortal beings } \quad C_{2} \text { : men } \quad C_{3} \text { : Greeks } \\
& s_{1} \text { : Some } C_{2} \text { are not } C_{3} . \quad S_{a}\left(C_{2}\right)=S_{b}\left(\mathbb{C}_{3}\right) \\
& s_{2}: \text { All } C_{2} \text { are } C_{1} . \quad C_{2}=S_{c}\left(C_{1}\right) \quad(2) ; \quad \stackrel{+}{C}_{1}=S_{d}\left(\stackrel{C}{C}_{2}\right) \\
& \therefore s_{3} \text { : Some } C_{1} \text { are not } C_{3} . \quad S_{x}\left(C_{1}\right)=S_{y}\left(\stackrel{C}{C}_{3}\right)
\end{aligned}
$$

Set equality (1) is selected because it includes a subset of $C_{2}$ as one of its members, and (2) is selected because it includes $C_{2}$ as one of its members.

$$
\begin{aligned}
S_{a}\left(C_{2}\right) & =S_{b}\left(\overleftarrow{C}_{3}\right) \\
C_{2} & =S_{c}\left(C_{1}\right)
\end{aligned}
$$

In (1), $C_{2}$ is replaced by the right-hand member of equality (2):

$$
S_{a}\left(S_{c}\left(C_{1}\right)\right)=S_{e}\left(C_{1}\right)=S_{b}\left(\stackrel{+}{C}_{3}\right)
$$

From (1) and (2), the following equality was deduced:

$$
S_{e}\left(C_{1}\right)=S_{b}\left(\overleftarrow{C}_{3}\right)
$$

If in (3) subscripts $x$ and $y$ are replaced by $e$ and $b$, respectively, equality (3) - an expression of the conclusion - is identical to equality (4), which was deduced from equalities (1) and (2), expressions of the first and second premises, respectively. Therefore, the syllogism considered is valid.

\section{Example 7}

$s_{1}$ : All engineers are pragmatic.

$s_{2}$ : Some engineers are wealthy.

$\therefore s_{3}$ : Some wealthy persons are pragmatic.

$C_{1}$ : wealthy persons $\quad C_{2}$ : engineers $\quad C_{3}$ : pragmatic persons

$$
\begin{aligned}
s_{1} \text { : } & \text { All } C_{2} \text { are } C_{3} . & C_{2} & =S_{a}\left(C_{3}\right) \\
s_{2} \text { : } & \text { Some } C_{2} \text { are } C_{1} . & S_{c}\left(C_{2}\right) & =S_{d}\left(C_{1}\right) \\
\therefore s_{3} & \text { : Some } C_{1} \text { are } C_{3} . & S_{x}\left(C_{1}\right) & =S_{y}\left(C_{3}\right)
\end{aligned}
$$


The premise $s_{2}$ "Some $C_{2}$ are $C_{1}$ " can also be expressed as "Some $C_{1}$ are $\mathrm{C}_{2}$ ". In that case it can be noted that the symbolic expressions of the categorical propositions $s_{1}, s_{2}$ and $s_{3}$ of the syllogism in example 7 are the same, respectively, as the symbolic expressions of the categorical propositions $s_{1}, s_{2}$ and $s_{3}$ of the syllogism in example 3 . It was proven that the latter syllogism is valid. Therefore, the syllogism considered in example 7 is valid.

\section{Example 8}

$s_{1}$ : No intellectuals are superstitious.

$s_{2}$ : Some French persons are intellectuals.

$\therefore s_{3}$ : Some French persons are not superstitious.

$C_{1}$ : French persons $\quad C_{2}$ : intellectuals $\quad C_{3}$ : superstitious persons

$$
\begin{aligned}
& s_{1} \text { : No } C_{2} \text { are } C_{3} \text {. } \\
& C_{2}=S_{a}\left(\stackrel{+}{C}_{3}\right) \\
& \text { (1); } \quad C_{3}=S_{b}\left(\stackrel{+}{C}_{2}\right) \\
& s_{2} \text { : Some } C_{1} \text { are } C_{2} \text {. } \\
& S_{c}\left(C_{1}\right)=S_{d}\left(C_{2}\right) \\
& \therefore s_{3} \text { : Some } C_{1} \text { are not } C_{3} . \quad S_{x}\left(C_{1}\right)=S_{y}\left(\stackrel{+}{C}_{3}\right)
\end{aligned}
$$

The symbolic expressions of the categorical propositions $s_{1}, s_{2}$ and $s_{3}$ of the syllogism in example 8 are the same, respectively, as the symbolic expressions of the categorical propositions $s_{1}, s_{2}$ and $s_{3}$ of the syllogism in example 4 . It was proven that the latter syllogism is valid. Therefore, the syllogism considered in example 8 is valid.

\section{Example 9}

$s_{1}$ : All men are rational.

$s_{2}$ : All Spaniards are men.

$\therefore s_{3}$ : All Spaniards are rational.

$$
\begin{aligned}
& C_{1} \text { : Spaniards } \quad C_{2} \text { : men } \quad C_{3} \text { : rational men } \\
& s_{1}: \text { All } C_{2} \text { are } C_{3} . \quad C_{2}=S_{a}\left(C_{3}\right) \quad(1) ; \quad \overleftarrow{C}_{3}=S_{b}\left(\overleftarrow{C}_{2}\right) \\
& s_{2} \text { : All } C_{1} \text { are } C_{2} . \quad C_{1}=S_{c}\left(C_{2}\right) \quad(2) ; \quad \stackrel{+}{C}_{2}=S_{d}\left(\stackrel{+}{C}_{1}\right) \\
& \therefore s_{3} \text { : All } C_{1} \text { are } C_{3} . \quad C_{1}=S_{x}\left(C_{3}\right) \quad(3) ; \quad \vec{C}_{3}=S_{y}\left(\stackrel{+}{C}_{1}\right)
\end{aligned}
$$

Set equality (1) is selected because it includes $C_{2}$ as one of its members, and set equality (2) is selected because it includes a subset of $C_{2}$ as one of its members.

$$
\begin{aligned}
& C_{2}=S_{a}\left(C_{3}\right) \\
& C_{1}=S_{c}\left(C_{2}\right)
\end{aligned}
$$


In (2), $C_{2}$ is replaced by the right-hand member of equality (1):

$$
C_{1}=S_{c}\left(S_{a}\left(C_{3}\right)\right)=S_{e}\left(C_{3}\right)
$$

From (1) and (2), the following equality was deduced:

$$
C_{1}=S_{e}\left(C_{3}\right)
$$

If in (3) the subscript $x$ is replaced by $e$, equality (3) - an expression of the conclusion - is identical to equality (4), which was deduced from equalities (1) and (2), expressions of the first and second premises, respectively. Therefore, the syllogism considered is valid.

Another proof of validity of that syllogism is provided below.

Set equality $\left(1^{\prime}\right)$ is selected because it includes a subset of $\vec{C}_{2}$ as one of its members, and $\left(2^{\prime}\right)$ is selected because it includes $\bar{C}_{2}$ as one of its members.

$$
\begin{aligned}
& \overleftarrow{C}_{3}=S_{b}\left(\overleftarrow{C}_{2}\right) \\
& \overleftarrow{C}_{2}=S_{d}\left(\overleftarrow{C}_{1}\right)
\end{aligned}
$$

In $\left(1^{\prime}\right), \stackrel{+}{C}_{2}$ is replaced by the right-hand member of equality $\left(2^{\prime}\right)$ :

$$
\stackrel{+}{C}_{3}=S_{b}\left(S_{d}\left(\stackrel{+}{C}_{1}\right)\right)=S_{f}\left(\stackrel{+}{C}_{1}\right)
$$

From $\left(1^{\prime}\right)$ and $\left(2^{\prime}\right)$ the following equality was deduced:

$$
\stackrel{+}{C}_{3}=S_{f}\left(\stackrel{C}{C}_{1}\right)
$$

If in $\left(3^{\prime}\right)$ the subscript $y$ is replaced by $f$, equality $\left(3^{\prime}\right)$ - an expression of the conclusion - is identical to equality $\left(4^{\prime}\right)$, which was deduced from equalities $\left(1^{\prime}\right)$ and $\left(2^{\prime}\right)$, expressions of the first and second premises, respectively. Therefore, another proof of the validity of the syllogism considered has been obtained.

Example 10

$s_{1}:$ All sculptors are artists.

$s_{2}$ : No artists are fossils.

$\therefore s_{3}$ : No fossils are sculptors.

$$
\begin{array}{rrrr}
C_{1} \text { : fossils } & C_{2} \text { : artists } & & C_{3} \text { : sculptors } \\
s_{1}: \text { All } C_{3} \text { are } C_{2} . & C_{3}=S_{a}\left(C_{2}\right) & (1) ; & \vec{C}_{2}=S_{b}\left(\stackrel{+}{C}_{3}\right) \\
s_{2}: \text { No } C_{2} \text { are } C_{1} . & C_{2}=S_{c}\left(\stackrel{+}{C}_{1}\right) & (2) ; & C_{1}=S_{d}\left(\stackrel{+}{C}_{2}\right) \\
\therefore s_{3}: \text { No } C_{1} \text { are } C_{3} . & C_{1}=S_{x}\left(\stackrel{+}{C}_{3}\right) & (3) ; & C_{3}=S_{y}\left(\mathbb{C}_{1}\right)
\end{array}
$$


Set equality (1) is selected because it includes a subset of $C_{2}$ as one of its members, and set equality (2) is selected because it includes $C_{2}$ as one of its members.

$$
\begin{aligned}
& C_{3}=S_{a}\left(C_{2}\right) \\
& C_{2}=S_{c}\left(\stackrel{C}{C}_{1}\right)
\end{aligned}
$$

In (1), $C_{2}$ is replaced by the right-hand member of equality (2):

$$
C_{3}=S_{a}\left(S_{c}\left(\overleftarrow{C}_{1}\right)\right)=S_{e}\left(\stackrel{+}{C}_{1}\right)
$$

From (1) and (2), the following equality was deduced:

$$
C_{3}=S_{e}\left(\stackrel{+}{C}_{1}\right)
$$

If in $\left(3^{\prime}\right)$ the subscript $y$ is replaced by $e$, equality $\left(3^{\prime}\right)-$ an expression of the conclusion - is identical to equality (4), which was deduced from equalities (1) and (2), expressions of the first and second premises, respectively. Therefore, the syllogism considered is valid.

Another proof of validity of that syllogism is provided below.

Set equality $\left(1^{\prime}\right)$ is selected because it includes $\vec{C}_{2}$ as one of its members, and $\left(2^{\prime}\right)$ is selected because it includes a subset of $\stackrel{t}{C}_{2}$ as one of its members.

$$
\begin{aligned}
& \overleftarrow{C}_{2}=S_{b}\left(\grave{C}_{3}\right) \\
& C_{1}=S_{d}\left(\grave{C}_{2}\right)
\end{aligned}
$$

In $\left(2^{\prime}\right), \stackrel{+}{C}_{2}$ is replaced by the right-hand member of equality $\left(1^{\prime}\right)$ :

$$
C_{1}=S_{a}\left(S_{b}\left(\stackrel{\leftarrow}{C}_{3}\right)\right)=S_{f}\left(\overleftarrow{C}_{3}\right)
$$

From $\left(1^{\prime}\right)$ and $\left(2^{\prime}\right)$ the following equality was deduced:

$$
C_{1}=S_{f}\left(\stackrel{+}{C}_{3}\right)
$$

If in (3) the subscript $x$ is replaced by $f$, equality (3) - an expression of the conclusion - is identical to equality $\left(4^{\prime}\right)$, which was deduced from equalities $\left(1^{\prime}\right)$ and $\left(2^{\prime}\right)$, expressions of the first and second premises, respectively. Therefore, another proof of the validity of the syllogism considered has been obtained.

\section{Example 11}

$s_{1}$ : No humanists are corrupt.

$s_{2}$ : All despots are corrupt.

$\therefore s_{3}$ : No despots are humanists. 


$$
\begin{aligned}
& C_{1} \text { : despots } \quad C_{2} \text { : corrupt persons } \quad C_{3} \text { : humanists } \\
& s_{1}: \text { No } C_{3} \text { are } C_{2} . \quad C_{3}=S_{a}\left(\stackrel{+}{C}_{2}\right) \quad(1) ; \quad C_{2}=S_{b}\left(\stackrel{C}{C}_{3}\right) \\
& s_{2}: \text { All } C_{1} \text { are } C_{2} . \quad C_{1}=S_{c}\left(C_{2}\right) \quad(2) ; \quad \stackrel{+}{C}_{2}=S_{d}\left(\stackrel{+}{C}_{1}\right) \\
& \therefore s_{3}: \text { No } C_{1} \text { are } C_{3} . \quad C_{1}=S_{x}\left(\stackrel{+}{C}_{3}\right) \quad(3) ; \quad C_{3}=S_{y}\left(\stackrel{十}{C}_{1}\right)
\end{aligned}
$$

Set equality $\left(1^{\prime}\right)$ is selected because it includes $C_{2}$ as one of its members, and set equality (2) is selected because it includes a subset of $C_{2}$ as one of its members.

$$
\begin{aligned}
& C_{2}=S_{b}\left(\stackrel{+}{C}_{3}\right) \\
& C_{1}=S_{c}\left(C_{2}\right)
\end{aligned}
$$

In $(2), C_{2}$ is replaced by the right-hand member of equality $\left(1^{\prime}\right)$ :

$$
C_{1}=S_{c}\left(S_{b}\left(\stackrel{+}{C}_{3}\right)\right)=S_{e}\left(\stackrel{+}{C}_{3}\right)
$$

From $\left(1^{\prime}\right)$ and (2), the following equality was deduced:

$$
C_{1}=S_{e}\left(\stackrel{+}{C}_{3}\right)
$$

If in (3) the subscript $x$ is replaced by $e$, equality (3) - an expression of the conclusion - is identical to equality (4), which was deduced from equalities $\left(1^{\prime}\right)$ and (2), expressions of the first and second premises, respectively. Therefore, the syllogism considered is valid.

Another proof of validity of that syllogism is provided below.

Set equality (1) is selected because it includes a subset of $\stackrel{\leftarrow}{C}_{2}$ as one of its members, and $\left(2^{\prime}\right)$ is selected because it includes $\stackrel{+}{C}_{2}$ as one of its members.

$$
\begin{aligned}
& C_{3}=S_{a}\left(\stackrel{\mathrm{C}}{2}_{2}\right) \\
& \stackrel{\mathrm{C}}{2}_{2}=S_{d}\left({\stackrel{+}{C_{1}}}_{1}\right)
\end{aligned}
$$

In (1), $\vec{C}_{2}$ is replaced by the right-hand member of equality $\left(2^{\prime}\right)$ :

$$
C_{3}=S_{a}\left(S_{d}\left(\stackrel{C}{C}_{1}\right)\right)=S_{f}\left(\stackrel{C}{C}_{1}\right)
$$

From (1) and (2) the following equality was deduced:

$$
C_{3}=S_{f}\left(\stackrel{+}{C}_{1}\right)
$$

If in $\left(3^{\prime}\right)$ the subscript $y$ is replaced by $f$, equality $\left(3^{\prime}\right)-$ an expression of the conclusion - is identical to equality $\left(4^{\prime}\right)$, which was deduced from equalities (1) and $\left(2^{\prime}\right)$, expressions of the first and second premises, respectively. Therefore, another proof of the validity of the syllogism considered has been obtained. 


\section{Example 12}

$s_{1}$ : Some mammals are dogs.

$s_{2}$ : All mammals are vertebrates.

$\therefore s_{3}$ : Some vertebrates are dogs.

$$
\begin{array}{rrrrr}
C_{1} \text { : vertebrates } & C_{2} \text { : mammals } & & \multicolumn{1}{l}{C_{3} \text { : dogs }} \\
s_{1} \text { : Some } C_{2} \text { are } C_{3} . & S_{a}\left(C_{2}\right)=S_{b}\left(C_{3}\right) & (1) & \\
s_{2} \text { : All } C_{2} \text { are } C_{1} . & C_{2}=S_{c}\left(C_{1}\right) & (2) ; & { }_{C_{1}}=S_{d}\left(\stackrel{+}{C}_{2}\right) \\
\therefore s_{3} \text { : } & \text { Some } C_{1} \text { are } C_{3} . & S_{x}\left(C_{1}\right)=S_{y}\left(C_{3}\right) &
\end{array}
$$

Set equality (1) is selected because one of its members is a subset of $C_{2}$, and set equality (2) is selected because one of its members is $C_{2}$.

$$
\begin{aligned}
S_{a}\left(C_{2}\right) & =S_{b}\left(C_{3}\right) \\
C_{2} & =S_{c}\left(C_{1}\right)
\end{aligned}
$$

In (1), $C_{2}$ is replaced by the right-hand member of equality (2):

$$
S_{a}\left(S_{c}\left(C_{1}\right)\right)=S_{b}\left(C_{3}\right)
$$

From (1) and (2), the following equality was deduced:

$$
S_{e}\left(C_{1}\right)=S_{b}\left(C_{3}\right)
$$

If in (3) subscripts $x$ and $y$ are replaced by $e$ and $b$, respectively, equality (3) - an expression of the conclusion - is identical to equality (4), which was deduced from (1) and (2), expressions of the first and second premises, respectively. Therefore, the syllogism considered is valid.

Example 13

$s_{1}:$ No artists are Neo-Kantians.

$s_{2}$ : Some Germans are Neo-Kantians.

$\therefore s_{3}$ : Some Germans are not artists.

$$
\begin{aligned}
& C_{1} \text { : Germans } \quad C_{2} \text { : Neo-Kantians } \quad C_{3} \text { : artists } \\
& s_{1} \text { : No } C_{3} \text { are } C_{2} . \quad C_{3}=S_{a}\left(\stackrel{C}{C}_{2}\right) \quad(1) ; \quad C_{2}=S_{b}\left(\stackrel{C}{C}_{3}\right) \\
& s_{2} \text { : Some } C_{1} \text { are } C_{2} . \quad S_{c}\left(C_{1}\right)=S_{d}\left(C_{2}\right) \\
& \therefore s_{3} \text { : Some } C_{1} \text { are not } C_{3} . \quad S_{x}\left(C_{1}\right)=S_{y}\left(\stackrel{+}{C}_{3}\right)
\end{aligned}
$$

Set equality $\left(1^{\prime}\right)$ is selected because one of its members is $C_{2}$, and set equality (2) is selected because one of its members is a subset of $C_{2}$.

$$
\begin{aligned}
C_{2} & =S_{b}\left(\overleftarrow{C}_{3}\right) \\
S_{c}\left(C_{1}\right) & =S_{d}\left(C_{2}\right)
\end{aligned}
$$


In (2), $C_{2}$ is replaced by the right-hand member of equality $\left(1^{\prime}\right)$ :

$$
S_{c}\left(C_{1}\right)=S_{d}\left(S_{b}\left(\overleftarrow{C}_{3}\right)\right)=S_{e}\left(\overleftarrow{C}_{3}\right)
$$

From $\left(1^{\prime}\right)$ and $(2)$, the following equality was deduced:

$$
S_{c}\left(C_{1}\right)=S_{e}\left(\stackrel{+}{C}_{3}\right)
$$

If in (3) subscripts $x$ and $y$ are replaced by $c$ and $e$, respectively, equality (3) - an expression of the conclusion - is identical to equality (4), which was deduced from equalities $\left(1^{\prime}\right)$ and (2), expressions of the first and second premises, respectively. Therefore, the syllogism considered is valid.

\section{Example 14}

$s_{1}$ : All poets are visionaries.

$s_{2}$ : All prophets are visionaries.

$\therefore s_{3}$ : Some prophets are poets.

$C_{1}$ : prophets $\quad C_{2}$ : visionaries $\quad C_{3}$ : poets

$$
\begin{aligned}
& s_{1}: \text { All } C_{3} \text { are } C_{2} . \quad C_{3}=S_{a}\left(C_{2}\right) \quad(1) ; \quad \stackrel{+}{C}_{2}=S_{b}\left(\stackrel{+}{C}_{3}\right) \\
& s_{2}: \text { All } C_{1} \text { are } C_{2} . \quad C_{1}=S_{c}\left(C_{2}\right) \quad(2) ; \quad \stackrel{+}{C}_{2}=S_{a}\left(\mathbb{C}_{1}\right) \\
& \therefore s_{3} \text { : Some } C_{1} \text { are } C_{3} . \quad S_{x}\left(C_{1}\right)=S_{y}\left(C_{3}\right)
\end{aligned}
$$

It is not possible to select two set equalities such that a) one of them corresponds to one of the premises and one of the members is $C_{2}$, and b) the other equality corresponds to the other premise and one of its members is a subset $C_{2}$. In effect, requirement a) is not satisfied in any of the equalities corresponding to the premises; nor is it possible to select two set equalities such that c) one of them corresponds to one of the premises and one of its members is $\vec{C}_{2}$, and d) the other equality corresponds to the other premise and one of its members is a subset of $\vec{C}_{2}$. In effect, requirement d) is not satisfied in any of equalities corresponding to the premises. Therefore, this syllogism considered is not valid.

\section{Example 15}

$s_{1}$ : Some landowners are not egotists.

$s_{2}$ : No philanthropists are egotists.

$\therefore s_{3}$ : Some philanthropists are landowners.

$$
\begin{aligned}
& C_{1} \text { : philanthropists } \quad C_{2} \text { : egotists } \quad C_{3} \text { : landowners } \\
& s_{1} \text { : Some } C_{3} \text { are not } C_{2} . \quad S_{a}\left(C_{3}\right)=S_{b}\left(\stackrel{+}{C}_{2}\right) \\
& s_{2}: \text { No } C_{1} \text { are } C_{2} . \quad C_{1}=S_{c}\left(\stackrel{+}{C}_{2}\right) \\
& \therefore s_{3} \text { : Some } C_{1} \text { are } C_{3} . \quad S_{x}\left(C_{1}\right)=S_{y}\left(C_{3}\right) \\
& \text { (2); } \quad C_{2}=S_{d}\left(\stackrel{+}{C}_{1}\right)
\end{aligned}
$$


It is not possible to select two set equalities such that a) one of them corresponds to one of premises and one of its members is $C_{2}$, and b) the other equality corresponds to the other premise and one of its members is a subset of $C_{2}$. In effect, requirement a) is satisfied in equality $\left(2^{\prime}\right)$ corresponding to the second premise, but requirement b) is not satisfied in equality (1) corresponding to the first premise which does not have a member that is a subset of $C_{2}$. Nor is it possible to select two set equalities such that c) one of them corresponds to one of the premises and one of its members is $\vec{C}_{2}$, and d) the other equality corresponds to the other premise and one of its members is a subset of $\vec{C}_{2}$. In effect, requirement $\mathrm{c}$ ) is not satisfied in any of equalities corresponding to the premises because $\vec{C}_{2}$ is not a member of any of them. Therefore, the syllogism considered is not valid.

Example 16

$s_{1}$ : Some philosophers are humanists.

$s_{2}$ : No humanists are egotists.

$\therefore s_{3}$ : Some philosophers are egotists.

$$
\begin{array}{rrrr}
C_{1} \text { : philosophers } & C_{2} \text { : humanists } & & C_{3} \text { : egotists } \\
s_{1} \text { : } & \text { Some } C_{1} \text { are } C_{2} . & S_{a}\left(C_{1}\right)=S_{b}\left(C_{2}\right) & (1) \\
s_{2} \text { : No } C_{2} \text { are } C_{3} . & C_{2}=S_{c}\left(\mathbb{C}_{3}\right) & (2) ; & C_{3}=S_{d}\left(\mathbb{C}_{2}\right) \\
\therefore s_{3} \text { : } & \text { Some } C_{1} \text { are } C_{3} . & S_{x}\left(C_{1}\right)=S_{y}\left(C_{3}\right)
\end{array}
$$

Set equality (1) is selected because one of its members is a subset of $C_{2}$, and (2) is selected because one of its members is $C_{2}$.

$$
\begin{aligned}
S_{a}\left(C_{1}\right) & =S_{b}\left(C_{2}\right) \\
C_{2} & =S_{c}\left(\vec{C}_{3}\right)
\end{aligned}
$$

In (1), $C_{2}$ is replaced by the right-hand member of equality (2):

$$
S_{a}\left(C_{1}\right)=S_{b}\left(S_{c}\left(\stackrel{C}{C}_{3}\right)=S_{e}\left(\overleftarrow{C}_{3}\right)\right.
$$

From (1) and (2), the following equality was deduced:

$$
S_{a}\left(C_{1}\right)=S_{e}\left(\stackrel{+}{C}_{3}\right)
$$

If in (3) the subscripts $x$ and $y$ are replaced by $a$ and $e$, respectively, an equality which is identical or equivalent to (4) is not obtained. It is not possible to deduce equality (4), corresponding to the conclusion, from set equalities (1) and (2) corresponding to the first premise and second premise, respectively. Nor is it possible to select two set equalities such that a) one of them corresponds to one of the premises and one of its members is $\vec{C}_{2}$, and b) the other equality 
corresponds to the other premise and one of its members is a subset of $\vec{C}_{2}$. In effect, requirement b) is satisfied in equality $\left(2^{\prime}\right)$ corresponding to the second premise, but requirement a) is not satisfied because equality (1) corresponding

to the first premise does not include $\vec{C}_{2}$ as one of its members. Therefore, the syllogism considered is not valid.

\section{Discussion and Perspectives}

The authors are conducting a research program whose main objectives are the following:

(1) show the natural continuity existing in different logics - classical bivalent logic and diverse variants of several non-classical logics - among different calculi, such as propositional calculus and predicate calculus (expressed in terms of set theory).

(2) specify how classical logic can be viewed as a "limit case" of certain variants to be considered of several non-classical logics. To become familiar with what is meant by "limit case" and how this study is being implemented, see (CFL) [19].

One part of the tasks focusing on achieving objective (1) consists of presenting methods to evaluate the validity - or lack of it - of types of reasoning with different degrees of complexity. The possibility of automating those methods to use them in disciplines such as control engineering and artificial intelligence is of particular interest.

Categorical syllogisms are types of reasoning with a low level of complexity which have played an important role in logic, from historical and instructional standpoints. For this reason, the authors have chosen them as a preliminary "testbed" for different methods to determine the validity of types of reasoning such as those specified in this article, and such as those presented earlier [8], [9]. Of course, the complexity of these methods will increase when considering types of reasoning more complex than categorical syllogisms.

Future work will address the modification of the above methods so that not only will they be "instruments" to determine the validity - or lack of it - of different types of reasoning, but they will also be resources which will make possible the automatic generation of valid types of reasoning given different sets of data.

\section{References}

[1] Quine, W. V. (1952; 1982). Part II. General Terms and Quantifiers. Section 16. Syllogisms. Methods of Logic, 4th ed. Harvard University Press, 102-108.

[2] Moktefi, A. \& Shin, S.-J., eds. (2013). Visual Reasoning with Diagrams. Birkhäuser/Springer.

[3] Nakatsu, R. T. (2010). Diagrammatic Reasoning in AI. Wiley. 
[4] Anderson, M., B. Meyer \& Olivier, P., eds. (2002). Diagrammatic Representation and Reasoning. Springer.

[5] Sasakura, M. (2001). A Visualization Method for Knowledge Represented by General Logic Programs, Proceedings Fifth International Conference on Information Visualization (IEEE).

[6] Allwein, G. \& Barwise, J., eds. (1996). Logical Reasoning with Diagrams. Oxford University Press.

[7] Van Dyke, F. (1995). A Visual Approach to Deductive Reasoning, The Mathematics Teacher, 88, 6: 481-486, 492-494.

[8] Skliar, O., Monge, R. E., \& Gapper, S. (2015). Using Inclusion Diagrams as an Alternative to Venn Diagrams to Determine the Validity of Categorical Syllogisms. arXiv:1509.00926.

[9] Skliar, O., Gapper, S. \& Monge, R. E. (2021). Using the Membership Table Method (MTM) to Determine the Validity of Categorical Syllogisms. arXiv:2105.12251.

[10] Copi, I. M., Cohen, C. \& Rodych, V. (2019). Introduction to Logic, 15th ed. Routledge.

[11] Hurley, P. J. (2015), A Concise Introduction to Logic, 12th ed. Cengage Learning.

[12] Leary, C. C. \& Kristiansen, L. (2015). A Friendly Introduction to Mathematical Logic. Milne Library.

[13] Mendelson, E. (2009). Introduction to Mathematical Logic. CRC Press.

[14] Quine, W. V. (1952; 1982). Methods of Logic, 4th ed. Harvard University Press.

[15] Goldrei, D. (2017). Classical Set Theory: For Guided Independent Study. Chapman and Hall/CRC.

[16] Cunningham, D. W. (2016). Set Theory: A First Course. Cambridge University Press.

[17] Jech, T. (2013). Set Theory. Springer.

[18] Devlin, K. (1993). The Joy of Sets: Fundamentals of Contemporary Set Theory. 2nd ed. Springer.

[19] Skliar, O., Gapper, S. \& Monge, R. E. (2021). A Canonical Fuzzy Logic. arXiv:2105.12251. 